\title{
Research Progress of Non-drug Therapy of Traditional Chinese Medicine in the Treatment of Obese Polycystic Ovary Syndrome
}

\author{
Chanchan Li' ${ }^{1}$ Nan $\mathrm{Li}^{2 *}$ \\ ${ }^{1}$ Shaanxi University of Traditional Chinese Medicine, Xianyang 712000, Shaanxi Province, China \\ ${ }^{2}$ Affiliated Hospital of Shaanxi University of Traditional Chinese Medicine, Xianyang 712000, Shaanxi Province, China \\ *Corresponding author: Nan Li, linan_80_311@163.com
}

\begin{abstract}
Polycystic ovarian syndrome (PCOS) as a relatively common clinical reproductive endocrine disease, the incidence is increasing year by year and the treatment is difficult, among which obese PCOS accounts for nearly $50 \%$ of the total. In recent years, non-drug therapy of traditional Chinese medicine (TCM) has been effective in the treatment of PCOS. This article summarizes the relevant literature in the past ten years from acupuncture, acupoint embedding, moxibustion, and auricular points, and observes the effects of TCM non-drug therapy on endocrine and glucose and lipid metabolism in obese PCOS patients. The impact of various indicators such as body mass index, body mass index, etc., and discuss the problems in such treatments, as well as put forward suggestions to achieve the purpose of better guiding the clinical treatment of obese PCOS.
\end{abstract}

Keywords: Non-drug therapy of TCM; Obesity; Polycystic ovary syndrome; Review

Publication date: July 2021; Online publication: July 31, 2021

\section{Introduction}

Polycystic ovarian syndrome (PCOS) as a clinical common, reproductive endocrine diseases characterized by persistent anovulation and high androgen, mainly manifested as sparse or amenorrhea, obesity, infertility, hirsutism, polycystic ovaries, etc ${ }^{[1]}$. The disease is more common in adolescence and childbearing age Women, more than $50 \%$ of them are obese $\left(\mathrm{BMI} \geq 25 \mathrm{~kg} / \mathrm{m}^{2}\right)$, and abdominal obesity (waist circumference/hip circumference $\geq 0.80$ ) is the majority. The incidence of obese PCOS is increasing year by year, even as high as $6.5 \% \sim 8 \%{ }^{[2]}$, has become a major problem in women's lives, so the treatment of this disease is more urgent and important. Western medicine mainly uses symptomatic treatment to reduce weight, regulate menstrual cycles, reduce androgen levels, improve insulin sensitivity, and induce ovulation ${ }^{[3]}$, but the duration of the treatment is short, and there are certain side effects. For example, the ovulationstimulating drug clomiphene used in the treatment of PCOS can reduce Conception rate (30\% 35\%), side effects of increasing spontaneous abortion rate ${ }^{[4]}$, and long-term medication also adds to the economic burden of patients.

Obese PCOS belongs to the scope of "amenorrhea," "obesity," and "infertility" in TCM. At present, most of the common clinical PCOS belong to the type of spleen and kidney deficiency and phlegmdampness block ${ }^{[5]}$. The principle of TCM treatment of the disease is to replenish the spleen and kidney, remove dampness and resolve phlegm ${ }^{[6]}$. TCM has a long history of non-drug therapy. The treatment of obese PCOS has the advantages of high safety, fewer side effects, and low recurrence rate. It can improve the quality of life of PCOS patients, reduce social and economic burdens, and save medical expenses ${ }^{[7]}$. 
This article analyzes and summarizes the research progress of non-drug therapy in the treatment of obese PCOS with TCM in the past 10 years, in order to provide a certain reference for the diagnosis and treatment of this disease.

\section{Pinprick}

Dan Wu et al. ${ }^{[8]}$ Observation and treatment of 112 obese PCOS patients, 56 received oral metformin, and 56 received metformin combined with acupuncture, the acupuncture points are Zhongwan, Qihai, Xiawan, Bilateral Liangmen, Waterway, etc. The treatment time starts on the first day after menstruation is completed, and acupuncture is performed twice a week for three months. After treatment, the patient's ovarian volume (OV) was significantly reduced, the number of follicles was significantly reduced, body mass index (BMI), follicle stimulating hormone (FSH), luteinizing hormone (LH), testosterone (T) and the ratio of LH to FSH Leptin, serum leptin (LEP), etc. were significantly reduced, while estradiol (E2) was significantly increased, and the therapeutic effect of acupuncture was better than that of Western medicine alone. The difference between the two was statistically significant $(\mathrm{P}<0.05)$. This study shows that acupuncture therapy can not only reduce the weight of patients, but also improve their sex hormone levels and ovarian function.

\section{Acupoint catgut embedding}

Danjuan He et al. ${ }^{[9]}$ for 184 people obese patients with PCOS were treated with observation and treatment. 60 patients in the first group were treated with acupoint embedding, and 62 patients in the second group were given oral drospirenone and ethinyl estradiol tablets and metformin, for the remaining 62 patients, embedding and western medicine were given combined treatment. The acupoints for embedding are divided into two groups: the first group of acupoints is Zusanli, Guanyuan, Ganshu, Sanyinjiao, etc,the second group is Tianshu, Yinlingquan, Shenshu, and Fenglong. The embedding time should be selected outside the menstrual period, once a week, and the above three treatments last for three months. After treatment, the patient's body mass, BMI, waist circumference, hip circumference, and waist-to-hip ratio (WHR) decreased significantly $(\mathrm{P}<0.05)$, and the clinical symptoms were significantly improved. In addition, the patient had no obvious adverse reactions during the treatment process, indicating that this treatment method is relatively tolerable, the effect lasts for a long time, and the operation is relatively simple.

\section{Moxibustion}

Yuanyuan Han et al. ${ }^{[10]}$ observed and treated 50 obese PCOS patients, 25 of which were treated with acupuncture, and the remaining 25 were treated with acupuncture combined with medicinal moxibustion. The acupoints were selected from Baihui, Guanyuan, Uterus, Cilian, Taichong, Fenglong, etc. The medicine-separated moxibustion group performed medicine-separated moxibustion at Shenque and Guanyuan at the same time as acupuncture. The powder composition of medicine-separated moxibustion was as follows: atractylodes $15 \mathrm{~g}$, dried tangerine peel $15 \mathrm{~g}$, chuanxiong $10 \mathrm{~g}$, xiangfu $10 \mathrm{~g}$ and other TCM materials. Three times a week, medicine-separated moxibustion once a week, avoiding the first three days of menstruation. After three months, the patient's FSH value increased, and the LH, LH/FSH, and T values all decreased, indicating that combined acupuncture on the basis of drug-separated moxibustion has a significant effect on improving the patient's hormone-related indicators; after treatment, the patient's TCM symptom score, BMI, hirsutism and other clinical scores were all lower than before, indicating that medicine-separated moxibustion combined with acupuncture can significantly improve the patient's TCM symptoms, reduce their weight, and relieve their hirsutism symptoms. 


\section{Auricular point}

Yan Li et al. ${ }^{[11]}$ observed and treated 60 adolescents obese PCOS patients. Thirty patients were treated with ear acupuncture pressure on the spleen, stomach, large intestine, endocrine, triple burn, internal genitalia, and hunger points. One ear acupuncture point was applied to Wangbuxingzi, and then alternated with the other side after a week. Instruct the patient to rub the ear points before meals and half an hour before going to bed, $5 \mathrm{~min} / \mathrm{time}$, for 3 consecutive months. The remaining 30 were given lifestyle guidance, including psychological counseling, dietary taboos, and exercise guidance. After 3 months of treatment, the BMI, LH, LH/FSH, FINS levels and HOMA-IR of the patients treated with ear acupoints were significantly reduced. This study shows that ear-point pressing seeds can significantly reduce the patient's BMI, improve their reproductive endocrine function, and there is no obvious adverse reaction.

\section{Discussion}

There are many ways to treat obese PCOS with TCM non-drug therapy. It has important clinical effects in reducing the patient's body mass index, improving the level of reproductive endocrine and glucose and lipid metabolism. It is simple to operate, economical and has few side effects. It is easy for patients to accept, but still There are some problems:

(1) The etiology and pathogenesis of PCOS are still unclear, and its symptomology has no uniform conclusion. The syndrome types are complex and diverse and subjective.

(2) In TCM non-drug therapy, the rule of point selection, curative effect determination, and course selection are still lacking in scientific and standard nature.

(3) Related clinical trials have problems such as small sample size, small scope, time limitation and low standardization level.

(4) The long-term efficacy follow-up is not in place, and there is little data support in the statistics of its recurrence rate and adverse reactions.

In view of the existing problems, in the future, we should:

(1) Develop systematic PCOS syndrome standards to facilitate clinical application.

(2) The experimental research on obese PCOS should carry out large-scale and large-scale investigation and research to increase its reliability.

Carry out long-term follow-up of patients, closely follow up the development of their condition, and comprehensively conduct further research on the TCM non-drug therapy of PCOS from various aspects.

\section{Funding}

National Natural Science Foundation of China (81603656); Infertility Innovation Team (132041929); National Major and Intractable Disease Infertility Clinical Collaboration Pilot Project of Traditional Chinese and Western Medicine.

\section{Disclosure statement}

The author declares no conflict of interest.

\section{References}

[1] Yao HP, Yao GG, 2019, Progress in the Treatment of Polycystic Ovary Syndrome with Traditional Chinese Medicine. Western Journal of Traditional Chinese Medicine, 32(12): 144-147.

[2] Qiao J. Polycystic Ovary Syndrome, 2009, Peking University Medical Press, Beijing, 20-21

[3] He YL, Xu XJ, Li WJ, et al., 2015, Research Progress in Traditional Chinese and Western Medicine 
for Obese Polycystic Ovary Syndrome. Chinese Journal of Traditional Chinese Medicine, 33(07): 1614-1616.

[4] He P, Hu XY, 2006, The Current Situation of the Side Effects of Clomiphene Ovulation Induction and the Improvement Prospects of Integrated Traditional Chinese and Western Medicine. Journal of Jiangxi University of Traditional Chinese Medicine, (05): 74-76.

[5] Zhang YQ, 2017, The Effect of Acupuncture on Adiponectin, Leptin and Insulin Resistance in Obese Patients with Polycystic Ovary Syndrome. Chinese Medical Journal, 32(11): 2259-2262.

[6] He F, Chen WX, Li H, 2016, Discussion on the Relationship between Obesity and Polycystic Ovary Syndrome and Research Progress in the Treatment of Obese Polycystic Ovary Syndrome. Hebei Journal of Traditional Chinese Medicine, 38(9): 1422-1426, 1431. DOI:10.3969/j.issn.10022619.2016.09.038.

[7] Wu XK, Chang H, Zhang Y, et al., 2010, Progress in the Epidemiological Investigation of Polycystic Ovary Syndrome. Science \& Technology Review, 28(21): 101-105.

[8] Wu D, Wang XB, Cong HF, et al., 2020, The effect of Acupuncture on Ovarian Function in Obese Patients with Polycystic Ovary Syndrome. World Traditional Chinese Medicine, 15(16): 2482-2485.

[9] He DJ, Liang SR, Huang XT, 2020, Acupoint Catgut Implantation in the Treatment of Obese Polycystic Ovary Syndrome. Hubei Journal of Traditional Chinese Medicine, 42(02): 12-16.

[10] Han YY, 2018, Preliminary Observation on the Clinical Efficacy of Medicine-Separated Moxibustion and Acupuncture in the Treatment of Phlegm-Dampness Type Polycystic Ovary Syndrome. Shandong University of Traditional Chinese Medicine.

[11] Li Y, Hao SL, Zhang CL, et al., 2018, Clinical Observation on the Treatment of Adolescent Overweight/Obese Polycystic Ovary Syndrome by Pressing Seeds at Ear Points. Modern Journal of Integrated Traditional Chinese and Western Medicine, 27(35): 3877-3879+3906. 